The Astrophysical Journal, 492:281-285, 1998 January 1

(C) 1998. The American Astronomical Society. All rights reserved. Printed in U.S.A.

\title{
GRAVITATIONAL WAVES FROM PHASE TRANSITIONS OF ACCRETING NEUTRON STARS
}

\author{
K. S. CHENG ${ }^{1}$ AND Z. G. DAI ${ }^{2}$ \\ Received 1996 December 31; accepted 1997 August 6
}

\begin{abstract}
We propose that when neutron stars in low-mass X-ray binaries accrete sufficient mass and become millisecond pulsars, the interiors of these stars may undergo phase transitions, exciting stellar radial oscillations. We show that the radial oscillations will be mainly damped by gravitational-wave radiation instead of internal viscosity. Such gravitational waves may be detected by the advanced Laser Interferometer Gravitational-wave Observatory.
\end{abstract}

Subject headings: dense matter - gravitation — pulsars: general — stars: neutron — stars: oscillations

\section{INTRODUCTION}

Gravitational-wave astronomy may soon become an observational science, since three gravitational-wave experiments, including the Laser Interferometer Gravitationalwave Observatory (LIGO; Abramovici et al. 1992), are under construction. In astrophysics, neutron stars are widely believed to be the most promising source of gravitational radiation (for detailed reviews see Thorne 1987, 1995), which may provide a signature for the features of supernova explosion from the collapse of cores of massive stars (Burrows \& Hayes 1996); reveal the physics of stellar interiors from starquakes of pulsars (Zimmermann \& Szedenits 1979); and give information about the equation of state (EOS) of nuclear matter at high densities from mergers of binary neutron stars (Shibata, Nakamura, \& Oohara 1992, 1993; Rasio \& Shapiro 1994; Zhuge, Centrella, \& McMillan 1994; Davies et al. 1994; Ruffert, Janka, \& Schäfer 1996). We propose a new possible origin of gravitational-wave bursts: when neutron stars in low-mass $\mathrm{X}$-ray binaries accrete sufficient mass and become millisecond pulsars, the interiors of these stars can undergo phase transitions that excite stellar radial oscillations, producing strong gravitational-wave bursts.

\section{THE MODEL}

\subsection{Evolution of Neutron Stars in Low-Mass $X$-Ray Binaries}

According to the standard scenario for the evolution of low-mass X-ray binaries (Bhattacharya \& van den Heuvel 1991), mass is transferred from the companion to the neutron star, which is spun up to a millisecond period. The mass transfer from the companion drives the two stars apart, while processes such as orbital gravitational radiation or magnetic braking drive them together. These phenomena keep the system in a steady mass transfer state throughout the evolutionary timescale of the companion while the accretion rate of the neutron star is near the Eddington value. Thus, the neutron star can accrete a mass $\geq 0.5 M_{\odot}$ in $\sim 10^{8} \mathrm{yr}$, becoming a millisecond pulsar (van den Heuvel \& Bitzaraki 1995a, 1995b). If we assume that the masses of neutron stars before accretion are $1.4 M_{\odot}$ (which is supported by the current theories of Type II supernova explosion and observations of masses of pulsars [e.g.,

\footnotetext{
${ }^{1}$ Department of Physics, University of Hong Kong, Pokfulam Road, Hong Kong.

${ }^{2}$ Department of Astronomy, Nanjing University, Nanjing 210093,
}

the Hulse-Taylor binary system]), then the stars in an evolutionary timescale $\geq 10^{8}$ yr must become relatively massive $\left(\geq 1.8 M_{\odot}\right)$. This raises the question of what could possibly occur in the interiors of these massive neutron stars.

To study this question, we first analyze possible EOSs for neutron stars. So far there have been many approaches to determine an EOS for dense matter through the many-body theory of interacting hadrons. Unfortunately, these approaches have yielded EOSs with different stiffnesses, indicating very different structures for neutron stars. However, the EOSs should be constrained by the following two observations: First, Link, Epstein, \& van Riper (1992) used a model-independent approach to analyze the postglitch recovery in four isolated pulsars (Crab, Vela, PSR $0355+54$, and PSR $0525+21$ ) that are likely to be isolated $1.4 M_{\odot}$ neutron stars, and concluded that soft EOSs at high densities are ruled out. More detailed analyses of the postglitch curves of the Crab and Vela pulsars also draw similar conclusions (Alpar et al. 1993, 1994). Second, if the EOSs in cores of neutron stars with mass $\sim 1.4 M_{\odot}$ were soft, the massive compact objects after the accretion phase of lowmass X-ray binaries could be black holes (Brown 1988). In fact, these objects have been identified as millisecond pulsars. This means that soft EOSs are unlikely to occur in neutron stars with mass $\sim 1.4 M_{\odot}$. For these two reasons, we can assume that the EOS in neutron stars with mass $\sim 1.4 M_{\odot}$ is moderately stiff to stiff.

The above assumption is consistent with recent theoretical studies of the EOS for dense matter at high densities. First, because of the strong repulsion between nucleons and nucleon holes in the spin-isospin interaction, pion condensation is unlikely in neutron stars (Brown et al. 1988; Baym 1991). Second, the possibility of kaon condensation in dense matter has been suggested by Kaplan \& Nelson (1986), who assert that the energy of a negative kaon is lowered by interaction with nucleons. In neutron star matter in beta equilibrium, one expects negative kaons to be present if the energy to create one kaon in the matter is less than the electron chemical potential. However, Pandharipande, Pethick, \& Thorsson (1995) studied kaon-nucleon and nucleon-nucleon correlations in kaon condensation in dense matter and found the kaon energy is much larger than indicated by previous studies (see, e.g., Brown et al. 1992, 1994; Thorsson, Prakash, \& Lattimer 1994; Lee et al. 1995; Thorsson \& Wirzba 1995). On the other hand, if we adopt the electron chemical potentials from the simple 
parameterized models of Prakash, Ainsworth, \& Lattimer (1988), which represent a number of more realistic models, and compare these potentials with the kaon energies of the Hartree calculations (Pandharipande et al. 1995) in Figure 1 , then we can see that the density for kaon condensation is $\sim(5-7) \rho_{0}$ (where $\rho_{0}$ is the nuclear density), which significantly exceeds the central densities of neutron stars with mass $\sim 1.4 M_{\odot}$ and moderately stiff to stiff EOSs. Several authors (e.g., Ellis, Knorren, \& Prakash 1995; Schaffner \& Mishustin 1996; Dai \& Cheng 1997) have used the relativistic mean field approach to study kaon energies in neutron star matter and have also drawn similar conclusions. Third, it is thought that the density for deconfinement of nuclear matter to two-flavor quark matter is near (6-9) $\rho_{0}$ (Baym 1991). Therefore, the recent theoretical studies of dense matter also indicate that pion (or kaon) condensation or quark matter is unlikely to occur in neutron stars with mass $\sim 1.4 M_{\odot}$.

We now turn to the discussion of what happens in neutron stars with moderately stiff to stiff EOSs when they accrete mass $\sim 0.5 M_{\odot}$, viz., when their masses increase from 1.4 to about $1.8 M_{\odot}$. It is possible that the central densities of these massive stars are $\sim(5-7) \rho_{0}$ (see, e.g., Wiringa, Fiks, \& Fabrocini 1988). Once this condition is reached, several physical processes will take place in the stars. When $e^{-} \rightarrow K^{-}+v_{e}$, kaon condensation occurs spontaneously in the stellar interior. The appearance of this new phase destroys the hydrostatic equilibrium of the star because of the softening of the EOS of the core matter. This implies a structural transition of the whole star into a new, stable configuration with a condensed core of the new phase. The structural transition-neutron star corequakeoccurs on a dynamical timescale of milliseconds (Haensel \& Prószyński 1982; Fujii et al. 1996), in which the interior temperature may increase to $\sim 10 \mathrm{MeV}$ as a result of both the rapid compression of dense matter (Haensel, Denissov, $\&$ Popov 1990) and the energy released by the reaction $e^{-} \rightarrow K^{-}+v_{e}$. In the following we wish to add two

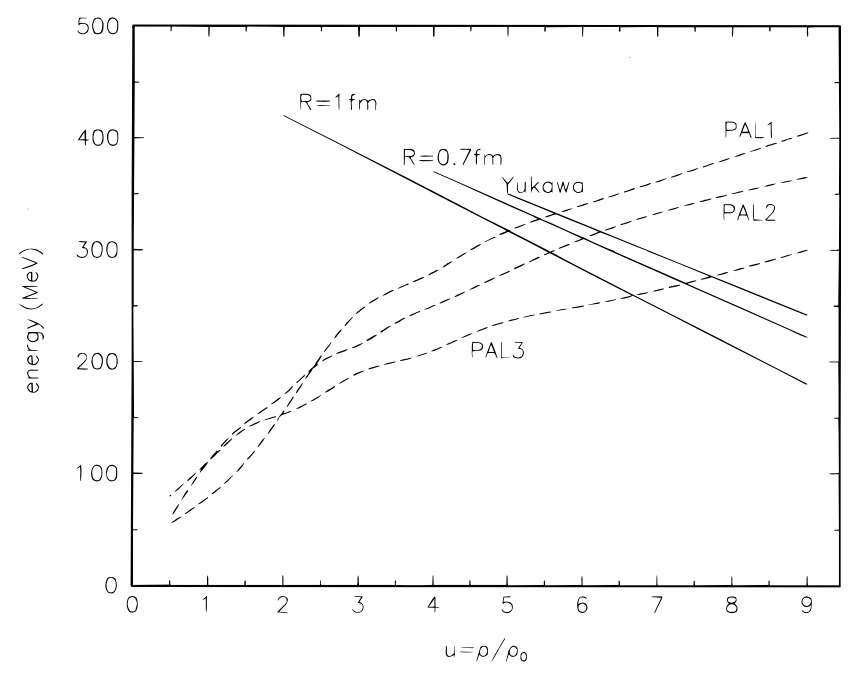

FIG. 1.-Energy of a single negative kaon in neutron star matter and electron chemical potential as functions of density. The three solid lines are kaon energies from the Hartree calculations of Pandharipande, Pethick, \& Thorsson (1995) for square wells of radii $R=1$ and $0.7 \mathrm{fm}$, and for a Yukawa potential. The dashed lines labeled "PAL1," "PAL2," and "PAL3" represent electron chemical potentials calculated from the parameterized models of Prakash, Ainsworth, \& Lattimer (1988), corresponding to three different forms of $F(u)$, which parameterizes the potential contribution to the symmetry energy. remarks. First, the phase transition discussed here is different from that in a hot nascent neutron star. The fully dynamical calculation of Baumgarte et al. (1996) shows that the latter is gradual, and the timescale for the phase transition to a black hole is about $100 \mathrm{~ms}$. In the case studied by these authors, a newly formed neutron star with baryonic mass of $1.78 M_{\odot}$ (which is slightly larger than the maximum baryonic mass $\left[1.77 M_{\odot}\right]$ for a deleptonized star with the hyperonic EOS of Glendenning [1985]) will be initially stable because of rich leptons. However, as the deleptonization proceeds through the emission of electron neutrinos, more and more hyperons are formed, and the EOS softens. This gradually reduces the mass limit for stability, and eventually the star will trigger gravitational instability and collapse to a black hole. During the collapse, the central temperature will increase from 60 to $\sim 100 \mathrm{MeV}$. Therefore, it can be seen that the strong lepton pressure and thermal pressure delay the phase transition of the hot star. For a cold star, as shown by Haensel \& Prószyński (1982), Haensel et al. (1990), and Fujii et al. (1996), the timescale for its phase transition to a meson-condensated star is about the free-fall time because of the absence of strong lepton pressure and thermal pressure. Thus, the phase transition of a cold star can be thought to be spontaneous. Second, the maximum mass of a kaon-condensated star depends on the critical density for kaon condensation. The larger the critical density, the larger the maximum mass (Fujii et al. 1996). If kaon condensation sets in at a density of $\sim 3 \rho_{0}$, as found by Brown et al. (1992), the maximum mass is about $1.5 M_{\odot}$, and a $1.4 M_{\odot}$ star after accreting more than 0.1 solar mass will collapse to a black hole (Brown \& Bethe 1994). If kaon condensation starts to occur at a density of (5-7) $\rho_{0}$, it is expected that the maximum mass of a kaon-condensated star may be larger than $1.8 M_{\odot}$. Hence, a $1.4 M_{\odot}$ star, after accreting $0.5 M_{\odot}$ mass and undergoing a phase transition, may still be stable against collapse to a black hole.

Alternatively, if the central nuclear matter in an accreting neutron star is deconfined into two-flavor quark matter, the quark matter will convert to three-flavor quark matter, because strange matter is thought to be more stable than nuclear matter. Thus a strange-matter seed is formed in the interior, and subsequently the strange matter will begin to swallow the neutron matter in the surroundings. This process should proceed in a timescale on the order of tens of milliseconds as a result of a detonation mode, and the interior temperature increases to $\geq 10 \mathrm{MeV}$ because the chemical energy of the two-flavor quark matter is dissipated into thermal energy (Dai, Peng, \& Lu 1995). The conversion of neutron stars to strange stars has been suggested as a possible origin of cosmological $\gamma$-ray bursts (Cheng \& Dai 1996). Furthermore, the phase transitions of massive neutron stars to either stars with kaon-condensation cores or strange stars can stimulate stellar radial oscillations. In the next subsection we will show that these newly born compact objects may be a strong source of gravitationalwave radiation if their rotation periods are of the order of milliseconds.

\subsection{Damping of Radial Oscillations}

It is well known that spherically symmetric oscillations of a nonrotating compact star do not radiate gravitational waves. However, if such a star is given a uniform rotation, the spherical symmetry of the system will be destroyed (Chau 1967). In this case, the oscillations are altered both 
because the equilibrium shape is changed and because the pulsation equations include the centrifugal and Coriolis force terms. The rotation thus makes the stellar radial oscillations produce gravitational waves, as proved explicitly by Chau (1967). Therefore, the radial oscillations discussed in the previous subsection are damped not only by dissipation of the vibration energy of stellar matter into heat, but also as a result of the conversion of this energy into gravitational-wave radiation. We first focus on the case in which a massive neutron star undergoes a phase transition to a star containing a kaon-condensation core. After $e^{-} \rightarrow K^{-}+v_{e}$, electron neutrinos are trapped in the interior and form an ideal Fermi-Dirac gas with $\mu_{v} \gg k T$ (where $T$ is the interior temperature), because the neutrino mean free path is much less than the stellar radius for $k T \sim 10 \mathrm{MeV}$. Subsequent evolution of the newborn star, which is analogous to that of a protoneutron star formed from supernova explosion (Burrows \& Lattimer 1986), can be divided into three stages. We neglect gravitational radiation. The first stage is deleptonization, whose timescale $\left(\tau_{d 1}\right)$ is of the order of $1 \mathrm{~s}$ (Sawyer \& Soni 1979), in which the interior temperature may increase significantly. In this stage, the radial oscillations are damped through the following reaction:

$$
\tilde{p}+e^{-} \leftrightarrow \tilde{n}+v_{e},
$$

where $\tilde{p}$ and $\tilde{n}$ represent quasiprotons and quasineutrons, respectively. Using the reaction matrix element of Brown et al. (1988), we have derived the net rate per baryon for this reaction at nonequilibrium,

$$
\begin{aligned}
\Gamma_{1}= & \frac{1}{24 \pi^{5} \hbar^{10} c^{5}} G_{\mathrm{F}}^{2} \cos ^{2} \theta_{\mathrm{C}} \cos ^{2}\left(\frac{\theta}{2}\right)\left(1+3 g_{A}^{2}\right) \\
& \times m_{n}^{*} m_{p}^{*} \mu_{e} \mu_{v}^{2} \Delta \mu\left[\Delta \mu^{2}+(2 \pi k T)^{2}\right],
\end{aligned}
$$

where $G_{\mathrm{F}}$ is the weak-coupling constant, $\theta_{\mathrm{C}}$ is the Cabibbo angle, and $\theta$ is the chiral angle for kaon condensation $\left(\theta \leq 60^{\circ}\right.$; Brown et al. 1994), $g_{A}$ is the Gamow-Teller coupling constant, $m_{n}^{*}$ and $m_{p}^{*}$ are the effective nucleon masses, and $\Delta \mu=\mu_{p}+\mu_{e}-\mu_{n}-\mu_{v}$ with $\mu_{i}$ being the chemical potential of particle $i$. In deriving equation (2), we have assumed $k T / c \ll\left|\boldsymbol{P}_{v}\right| \ll\left|\boldsymbol{P}_{n}\right|$, with $\boldsymbol{P}_{v}$ and $\boldsymbol{P}_{n}$ being the neutrino and neutron momenta, respectively. As the steps shown by Dai \& $\mathrm{Lu}$ (1996), using this reaction rate, we have further derived the bulk viscosity as

$$
\begin{aligned}
\eta_{1} \simeq & 1.8 \times 10^{25} \cos ^{-2}\left(\frac{\theta}{2}\right) Y_{e}^{-1 / 3} Y_{v}^{2 / 3} Y_{n}^{4 / 3} \\
& \times\left(\frac{\rho}{\rho_{0}}\right)^{5 / 3}\left(\frac{k T}{1 \mathrm{MeV}}\right)^{-2} \mathrm{~g} \mathrm{~cm}^{-1} \mathrm{~s}^{-1},
\end{aligned}
$$

where $Y_{e}, Y_{v}$, and $Y_{n}$ are the particle concentrations and $\rho$ is the stellar density. Therefore, the damping timescale (Sawyer 1980) is given by

$$
\tau_{v 1}=\rho R^{2} \eta_{1}^{-1} / 30,
$$

where $R$ is the stellar radius. Inserting equation (3) into this equation, we obtain

$$
\begin{aligned}
\tau_{v 1} \simeq & 0.52 \cos ^{2}\left(\frac{\theta}{2}\right) Y_{e}^{1 / 3} Y_{v}^{-2 / 3} Y_{n}^{-4 / 3} R_{6}^{2} \\
& \times\left(\frac{\rho}{\rho_{0}}\right)^{-2 / 3}\left(\frac{k T}{1 \mathrm{MeV}}\right)^{2} \mathrm{~s}
\end{aligned}
$$

where $R_{6}$ is in units of $10^{6} \mathrm{~cm}$. For the typical particle concentrations and $\rho \sim 6 \rho_{0}, R \sim 10^{6} \mathrm{~cm}$, and $k T \sim 10$ $\mathrm{MeV}$, we have $\tau_{v 1} \sim 12 Y_{e}^{1 / 3} Y_{v}^{-2 / 3} Y_{n}^{-4 / 3} \mathrm{~s}>12 \mathrm{~s} \gg \tau_{d 1}$.

In the second stage, the stellar interior has practically no trapped lepton number excess, as compared with catalyzed matter. The diffusion of $v_{e} \bar{v}_{e}$ is then driven by the temperature gradient. Locally, the equilibrium distribution function of $v_{e} \bar{v}_{e}$ can be approximated by the Fermi-Dirac function with a zero chemical potential. The neutrino diffusion timescale $\left(\tau_{d 2}\right)$ is of the order of $40 \mathrm{~s}$ (Sawyer \& Soni 1979). Using the analogy of deriving the bulk viscosity of (Haensel \& Schaeffer 1992), we obtain the damping timescale

$$
\tau_{v 2} \simeq 1.8 \times 10^{2} \cos ^{2}\left(\frac{\theta}{2}\right) Y_{e}^{-1 / 3} R_{6}^{2}\left(\frac{\rho}{\rho_{0}}\right)^{2 / 3} \mathrm{~s} .
$$

Clearly, $\tau_{v 2} \gg \tau_{d 2}$.

Third, after the neutrino diffusion, the temperature decreases to $\sim 1 \mathrm{MeV}$, and neutrinos can escape freely from the star. The damping timescale becomes

$$
\begin{aligned}
\tau_{v 3} \simeq & 2.6 \times 10^{-5} \cos ^{-2}\left(\frac{\theta}{2}\right) Y_{e}^{-1 / 3} R_{6}^{2} \\
& \times\left(\frac{\rho}{\rho_{0}}\right)^{2 / 3}\left(\frac{k T}{1 \mathrm{MeV}}\right)^{-4}\left(\frac{\omega}{10^{4} \mathrm{~s}^{-1}}\right)^{2} \mathrm{~s},
\end{aligned}
$$

where $\omega$ is the oscillation frequency. Therefore, we can conclude that the radial oscillations are not damped very efficiently by the bulk viscosity until the stage at which neutrinos escape freely.

We now consider gravitational radiation from rapidly spinning and oscillating neutron stars. The timescale for this process (Chau 1967) is

$$
\tau_{g} \simeq 0.41 M_{1.8}^{-1} R_{6}^{-2}\left(\frac{P}{2 \mathrm{~ms}}\right)^{4} \mathrm{~s},
$$

where $M_{1.8}$ is the stellar mass in units of $1.8 M_{\odot}$ and $P$ is the stellar rotation period. Here we have assumed that nucleons in the stellar interior are nonrelativistic and degenerate, and thus the adiabatic index is equal to $5 / 3$. When $\tau_{g} \leq \tau_{d 1}+\tau_{d 2}$, viz.,

$$
P \leq 6.2 \bar{\tau}_{d}^{1 / 4} M_{1.8}^{1 / 4} R_{6}^{1 / 2} \mathrm{~ms},
$$

where $\bar{\tau}=\left(\tau_{d 1}+\tau_{d 2}\right) /(40 \mathrm{~s})$, the gravitational radiation can damp the radial oscillations very efficiently. The frequency of the gravitational waves is equal to $\omega=2 \pi / \tau$, where $\tau$ is close to $5 \times 10^{-4} \mathrm{~s}$ for a typical neutron star model (Glass $\&$ Lindblom 1983; corrected for the gravitational redshift) with mass $M \sim 1.8 M_{\odot}$ and radius $R \sim 10^{6} \mathrm{~cm}$. the strength of the waves can be estimated using the quadrupole approximation to the Einstein field equations (Thorne 1987). This approximation shows that the gravitational strain is given by

$$
\begin{aligned}
h \simeq \frac{G}{c^{4}} \frac{\ddot{Q}}{r} \sim & 1.5 \times 10^{-23} R_{6}^{5}\left(\frac{\rho}{6 \rho_{0}}\right) \\
& \times\left(\frac{\alpha}{0.1}\right)\left(\frac{P}{2 \mathrm{~ms}}\right)^{-2}\left(\frac{r}{100 \mathrm{Mpc}}\right)^{-1},
\end{aligned}
$$

where $Q$ is the source's quadrupole moment (Chau 1967), $r$ is the distance of the source from Earth, and $\alpha$ is the relative oscillation amplitude $(=\delta R / R)$. The exact value of $\alpha$ is dependent upon both the EOS for pure nuclear matter and 
the EOS for kaon condensation. If the strength of the phase transitions $(\lambda)$, which is defined by the gap in the mass density between the onset and termination mass densities (Haensel \& Prószyński 1982), is larger than the critical value $\lambda_{c}=3 / 2$, then $\alpha$ can reach about $30 \%$ (Fujii et al. 1996). Here we assume that $\alpha$ is about 0.1 . Furthermore, the characteristic gravitational strain is

$$
\begin{aligned}
h_{c} \simeq h \sqrt{n} & \sim 4.3 \times 10^{-22} M_{1.8}^{-1 / 2} R_{6}^{4} \\
& \times\left(\frac{\rho}{6 \rho_{0}}\right)\left(\frac{\alpha}{0.1}\right)\left(\frac{\tau}{0.5 \mathrm{~ms}}\right)^{-1 / 2}\left(\frac{r}{100 \mathrm{Mpc}}\right)^{-1},
\end{aligned}
$$

where $n$ is the number of cycles of gravitational waves in the duration $\sim \tau_{g}$. The observed number of low-mass X-ray binaries in our Galaxy is $\sim 10^{2}$ (van Paradijs 1995), and thus the rate for phase transitions of their neutron stars is $\sim 10^{-6} \mathrm{yr}^{-1}$ because the typical accretion timescale is $\sim 10^{8}$ yr. An alternative estimation based on the number of millisecond pulsars and their lifetime also gives a similar value (Cheng \& Dai 1996). Therefore, the rate detected by the advanced LIGO detector (Abramovici et al. 1992) is estimated to be about three events per year.

Alternatively, neutron stars in low-mass X-ray binaries may accrete sufficient mass to convert into strange stars, as suggested by Cheng \& Dai (1996). In this case, first, the timescale for damping radial oscillations due to bulk viscosity is $\geq 10 \mathrm{~s}$ for a high temperature, $\geq 10 \mathrm{MeV}$ (Madsen 1992; Dai \& Lu 1996), so the gravitational radiation damping mechanism is also more efficient. Second, for the conversion of neutron stars with stiff EOSs to strange stars, the relative oscillation amplitude $(\alpha)$ may not be less than 0.1 . Third, the density for deconfinement may be larger than that for kaon condensation, and thus the oscillation frequency of strange stars is larger. If gravitational waves discussed in this work are observed by LIGO in the future, then observations, in principle, can distinguish between these two kinds of phase transitions. In addition, gravitational radiation for the case in which neutron stars convert to strange stars is likely to occur together with cosmological $\gamma$-ray bursts, because the fireballs formed during the conversion have very low baryon contamination (Cheng \& Dai 1996).

\section{SUMMARY AND DISCUSSION}

We have suggested that during the evolution of neutron stars in low-mass X-ray binaries the stars may undergo phase transitions to stars containing kaon condensation cores or strange stars when these neutron stars accrete sufficient mass from their companions. The phase transitions will excite stellar radial oscillations, which produce strong gravitational-wave bursts if the stellar rotation periods are of the order of milliseconds. The study of such gravitational radiation may provide information for the high-density EOS and the physics of phase transitions. In addition, the rate detected by the advanced LIGO is estimated to be about three events per year. Gravitational waves from colliding neutron stars must have a continuous spectrum, but waves from phase transitions of neutron stars appear to have a delta-function-like spectrum. This signature is expected to be confirmed by observations in the very near future.

However, we would like to point out that the physics at several times $\rho_{0}$ is still very controversial: therefore, if it exists, such phase transition is also not clear. Moreover, the existence of a new equilibrium configuration after the phase transition is also not certain. On the other hand, if we accept the current theories and use two different polytropic indices to approximate the EOS, there is evidence that in some cases no strong shocks are generated in the collapse process triggered by the phase transition, while in other cases (depending on the EOS before and after the transition) there could be strong shock-heating, causing the energy dissipation to be neutrino dominated, or even disrupt the whole star (W.-M. Suen \& A. Anninos 1997, private communication). Finally, we want to remark that the existence of such phase transition should be regarded as an assumption until the physics beyond several nuclear density is confirmed, e.g., by heavy-ion collision experiments, and the existence of a stable rotating and oscillating neutron star is only shown by numerical simulation and restricted to polytropic EOS.

We would like to thank the referee for helpful comments and suggestions. We also thank W. M. Suen and A. Anninos for bringing this problem to our attention and showing us their numerical results, W. Y. Chau for discussions of gravitational radiation, and Samuel Wong and M. C. Chu for discussion of equations of state at high densities. K. S. C. thanks an RGC grant of Hong Kong for support; Z. G. D. thanks the National Natural Science Foundation of China for support.

\section{REFERENCES}

Abramovici, A., et al. 1992, Science, 256, 325

Alpar, M. A., Chau, H. F., Cheng, K. S., \& Pines, D. 1993, ApJ, 409, 345

. 1994, ApJ, 427, L27

Baumgarte, T. W., Janka, H.-T., Keil, W., Shapiro, S. L., \& Teukolsky, S. A. 1996, ApJ, 468, 823

Baym, G. 1991, in Neutron Stars: Theory and Observation, ed. J. Ventura \& D. Pines (Dordrecht: Kluwer), 21

Bhattacharya, D., \& van den Heuvel, E. P. J. 1991, Phys. Rep., 203, 1

Brown, G. E. 1988, Nature, 336, 519

Brown, G. E., \& Bethe, H. A. 1994, ApJ, 423, 659

Brown, G. E., Kubodera, K., Page, D., \& Pizzochero, P. 1988, Phys. Rev. $\mathrm{D}, 37,2042$

Brown, G. E., Kubodera, K., Rho, M., \& Thorsson, V. 1992, Phys. Lett. B, 291,355

Brown, G. E., Lee, C.-H., Rho, M., \& Thorsson, V. 1994, Nucl. Phys. A, 567, 937

Burrows, A., \& Hayes, J. 1996, Phys. Rev. Lett., 76, 352

Burrows, A., \& Lattimer, J. M. 1986, ApJ, 307, 178

Chau, W. Y. 1967, ApJ, 147, 667

Cheng, K. S., \& Dai, Z. G. 1996, Phys. Rev. Lett., 77, 1210

Dai, Z. G., \& Cheng, K. S. 1997, Phys. Lett. B, 401, 219
Dai, Z. G., \& Lu, T. 1996, Z. Phys. A, 355, 415

Dai, Z. G., Peng, Q. H., \& Lu, T. 1995, ApJ, 440, 815

Davies, M. B., Benz, W., Piran, T., \& Thielemann, F. K. 1994, ApJ, 431, 742

Ellis, P. J., Knorren, R., \& Prakash, M. 1995, Phys. Lett. B, 349, 11

Fujii, H., Maruyama, T., Muto, T., \& Tatsumi, T. 1996, Nucl. Phys. A, 597, 645

Glass, E. N., \& Lindblom, L. 1983, ApJS, 53, 93

Glendenning, N. K. 1985, ApJ, 293, 470

Haensel, P., Denissov, A., \& Popov, S. 1990, A\&A, 240, 78

Haensel, P., \& Prószyński, M. 1982, ApJ, 258, 306

Haensel, P., \& Shaeffer, R. 1992, Phys. Rev. D, 45, 4708

Kaplan, D. B., \& Nelson, A. E. 1986, Phys. Lett. B, 175, 57

Lee, C.-H., Brown, G. E., Min, D.-P., \& Rho, M. 1995, Nucl. Phys. A, 585, 401

Link, B., Epstein, R. I., \& van Riper, K. A. 1992, Nature, 359, 616

Madsen, J. 1992, Phys. Rev. D, 46, 3290

Pandharipande, V. R., Pethick, C. J., \& Thorsson, V. 1995, Phys. Rev. Lett., 75,4567

Prakash, M., Ainsworth, T. L., \& Lattimer, J. M. 1988, Phys. Rev. Lett., 61, 2518

Rasio, F. A., \& Shapiro, S. L. 1994, ApJ, 432, 242 
Ruffert, M., Janka, H.-T., \& Schäfer, G. 1996, A\&A, 311, 532

Sawyer, R. F. 1980, ApJ, 237, 187

Sawyer, R. F., \& Soni, A. 1979, ApJ, 230, 859

Schaffner, J., \& Mishustin, I. N. 1996, Phys. Rev. C, 53, 1416

Shibata, M., Nakamura, T., \& Oohara, K. 1992, Prog. Theor. Phys., 88, 1079

1993, Prog. Theor. Phys., 89, 809

Thorne, K. S. 1987, in Three Hundred Years of Gravitation, ed. S. W. Hawking \& W. Israel (Cambridge: Cambridge Univ. Press), 330

. 1995, in Proc. Snowmass '94 Summer Study on Particle and Nuclear Astrophysics and Cosmology, ed. E. W. Kolb \& R. Peccei (Singapore: World Scientific), 1
Thorsson, V., Prakash, M., \& Lattimer, J. M. 1994, Nucl. Phys. A, 572, 693

Thorsson, V., \& Wirzba, A. 1995, Nucl. Phys. A, 589, 633

van den Heuvel, E. P. J., \& Bitzaraki, O. 1995a, A\&A, 297, L41

$1995 \mathrm{~b}$, in the Lives of the Neutron Stars, ed. M. A. Alpar, Ü. Kilzıloğlu \& J. van Paradijs (Dordrecht: Kluwer), 421

van Paradijs, J. 1995, in X-Ray Binaries, ed. W. H. G. Lewin, J. van Paradijs, \& E. P. J. van den Heuvel (Cambridge: Cambridge Univ. Press), 536

Wiringa, R. B., Fiks, V., \& Fabrocini, A. 1988, Phys. Rev. C, 38, 1010 Zhuge, X., Centrella, J. M., \& McMillan, S. L. M. 1994, Phys. Rev. D, 50, 6247

Zimmernam, M., \& Szedenits, E. 1979, Phys. Rev. D, 20, 351 